\title{
Association of high PDPN expression with pulmonary metastasis of osteosarcoma and patient prognosis
}

\author{
XINCHENG WANG ${ }^{1,2}$, WEI LI $^{2}$, JIAQI BI ${ }^{2}$, JIA WANG $^{3}$, LINYING NI ${ }^{4}$, QINGTAO SHI ${ }^{5}$ and QINGGANG MENG ${ }^{1,2}$ \\ ${ }^{1}$ Department of Orthopedics, The Fourth Affiliated Hospital of Harbin Medical University; ${ }^{2}$ Department of Orthopedics, \\ The First Hospital of Harbin City, Harbin, Heilongjiang 150010; Departments of ${ }^{3}$ Hepatobiliary Surgery, ${ }^{4}$ Orthopedics \\ and ${ }^{5}$ Pathology, The Affiliated Cancer Hospital of Harbin Medical University, Harbin, Heilongjiang 150081, P.R. China
}

Received August 14, 2018; Accepted May 31, 2019

DOI: $10.3892 / \mathrm{ol} .2019 .11053$

\begin{abstract}
Podoplanin (PDPN) is an important positive regulator of platelet aggregation and functions as a lymphatic endothelial marker. PDPN has been observed to be expressed in human tumor tissues and various cancer cell lines. In the present study, PDPN expression in patients with primary osteosarcoma was assessed at the mRNA and protein levels, and the associations between PDPN expression and pulmonary metastasis (PM) and prognosis were examined. Reverse transcription-quantitative PCR (RT-qPCR) analysis was used to detect the expression levels of PDPN in primary osteosarcoma tissues and paired normal bone tissues ( $n=20$ pairs). In addition, immunohistochemical analysis of PDPN expression was performed in 168 paraffin-embedded osteosarcoma tissue specimens and 23 matched normal tissues. The RT-qPCR results revealed higher mRNA expression levels of PDPN in patients with PM compared with patients without PM. Further survival analyses identified Enneking stage and PM as two independent prognostic indicators. Finally, univariate analysis revealed that high PDPN protein expression was significantly associated with Enneking stage and PM in patients with osteosarcoma.
\end{abstract}

\section{Introduction}

Osteosarcoma is a primary bone tumor originating in mesenchymal tissues, with a high prevalence and high rates of malignancy. The results of a survey in 2016 indicated that each year, 20,000-30,000 patients with osteosarcoma succumb to the disease globally, with the highest incidence rates $(70,000-80,000)$ among adolescents aged $15-19$ years (1).

Correspondence to: Dr Qinggang Meng, Department of Orthopedics, The Fourth Affiliated Hospital of Harbin Medical University, 37 Yi Yuan Street 37, Harbin, Heilongjiang 150010, P.R. China

E-mail: 13674677752@163.com

Key words: podoplanin, osteosarcoma, pulmonary metastasis, prognosis
While standard methods of treatment, including chemotherapy, palliative radiotherapy and limb amputation or sparing can be effective, there is a high probability (80-90\%) that pulmonary metastasis (PM) will develop $(1,2)$. PM is common in osteosarcoma and is an important prognostic indicator for patients. The five-year survival rate of patients with PM was 20\% in 2014, despite extensive research that has been undertaken to identify effective treatments for these patients $(3,4)$. The enhanced chest CT technique is a standard method used to evaluate PM in patients with osteosarcoma; however, the limited sensitivity of CT imaging can mean that PM is not detected during early stages (5). Currently, accurate and reliable pre-operative PM identification is not possible for patients with osteosarcoma, and a more detailed understanding of PM would be conducive to assessing patient prognosis, as well as to formulate effective treatment plans. Therefore, identification of PM in patients with osteosarcoma relies on future development of objective markers.

Encoded by the human PDPN gene, podoplanin is recognized as a type I transmembrane lymphoid glycoprotein (6). As a lymphatic endothelium marker, podoplanin is regulated by the lymphoid-specific homologous transformant gene, prospero homeobox 1. Podoplanin contains a platelet aggregation (PLAG)-inducing domain, which can activate the PLAG enzyme (6,7). Since PDPN induces platelet aggregation (8), it serves a crucial function in cell migration, as well as tumor cell dissemination $(9,10)$. Increased PDPN expression has been observed in a variety of human cancer cells $(11,12)$, and multiple studies have reported high PDPN expression in human osteosarcoma tissues (13) and various human osteosarcoma cell lines, including U2OS, HOS and MG63 (7). However, to the best of our knowledge, the association between PDPN expression and PM in patients with osteosarcoma has not yet been investigated, which was the primary focus of the present study.

\section{Materials and methods}

Ethics statement. The current study was performed in strict accordance with the Declaration of Helsinki. The Institutional Ethics Committees of Harbin Medical University (Harbin, China) and the Harbin Medical University Cancer Hospital (Harbin, China) approved the present study and written 
informed consent was obtained from patients directly or their legal guardians. All methods conformed to the associated institutional regulations and guidelines.

Cell line preparation and cultivation. The human fetal osteoblastic cell line, hFOB 1.19, and human osteosarcoma cell lines, MG63, Saos2, HOS and U2OS, were purchased from the American Type Culture Collection, and were maintained in a $5 \% \mathrm{CO}_{2}$ atmosphere at $37^{\circ} \mathrm{C}$. The cells were cultured in RPMI-1640 medium (Gibco; Thermo Fisher Scientific, Inc.) containing $10 \%$ fetal bovine serum (Gibco; Thermo Fisher Scientific, Inc.), $0.6 \%$ kanamycin sulfate (Gibco; Thermo Fisher Scientific, Inc.) and $1 \%$ antibiotic-antimycotic (Gibco; Thermo Fisher Scientific, Inc.). The medium was refreshed two or three times per week. Harvested cells were used for reverse transcription-quantitative PCR (RT-qPCR) analysis.

Patients and tissue specimens. A total of 20 pairs of fresh-frozen primary osteosarcoma tissue (POT) and adjacent non-cancerous bone tissue (NCBT) samples were obtained from patients undergoing resection surgery at the Department of Orthopedic Surgery, Among the 20 patients, 11 were male and 9 were female, with an average age of 21.5 years. Samples were obtained from patients at The Affiliated Cancer Hospital of Harbin Medical University between January 12017 and June 31 2017. The samples were used for RT-qPCR analysis.

Immunohistochemical (IHC) analyses were performed using 168 verified, paraffin-embedded osteosarcoma tissues collected from patients admitted to the Department of Orthopedics Surgery, Harbin Medical University Cancer Hospital between January 2003 and December 2012. These consisted of 98 male and 70 female patients (age range, 7-71 years; mean, 25.1 years). A total of 35 patients received preoperative anticancer treatment, and 23 patients exhibited synchronous distant PM. Clinicopathological parameters, including age, sex, maximum tumor diameter, Enneking stage (14), pre-operative serum alkaline phosphatase (ALP) levels and pre-operative PM status, were obtained from clinical and pathological records. Osteosarcoma was pathologically confirmed in all patients according to the World Health Organization bone tumor diagnosis and staging criteria (15). All patients were followed up until 31 December 2017 and monitored over a period of 1-126 months. Patients with whom contact was lost or had died from causes other than osteosarcoma were excluded. All patients included in the present study were still alive or were confirmed to have succumbed to the cause or complications associated with this disease until the follow-up deadline. Prognosis of patients was represented by statistics of patients who survived until the date of follow-up. For statistics of patient survival time, the unit was months.

$R N A$ extraction and $R T-q P C R$ analysis. TRIzol ${ }^{\circledR}$ reagent (Invitrogen; Thermo Fisher Scientific, Inc.) was used to extract total RNA from fresh osteosarcoma tissues and cell lines, according to the manufacturer's protocols. Total RNA was quantified by spectrophotometry analysis (Shimadzu Corporation). A universal cDNA synthesis kit (Toyobo Life Science) was employed to reverse transcribe RNA into cDNA, which was then analyzed by qPCR analysis using a SYBR Green PCR kit (Toyobo Life Science) and a Prism 7300 Sequence Detection system (Applied Biosystems; Thermo Fisher Scientific, Inc.). The thermocycling conditions were: Denaturation at $95^{\circ} \mathrm{C}$ for $30 \mathrm{sec}$; followed by 40 cycles of $95^{\circ} \mathrm{C}$ for $5 \mathrm{sec}$ and $60^{\circ} \mathrm{C}$ for $30 \mathrm{sec}$; and a final extension step of $95^{\circ} \mathrm{C}$ for $15 \mathrm{sec}, 60^{\circ} \mathrm{C}$ for $1 \mathrm{~min}, 95^{\circ} \mathrm{C}$ for $15 \mathrm{sec}$ and $60^{\circ} \mathrm{C}$ for $15 \mathrm{sec}$. The following primers were used: PDPN, forward 5'-AGCGAAGACCGCTATAAGTCTG-3' and reverse 5'-CTT TCTGAAGTTGGCAGATCCT-3'; GAPDH, forward 5'-GCA CCGTCAAGGCTGAGAAC-3' and reverse 5'-TGGTGAAGA CGCCAGTGGA-3'. GAPDH served as the reference gene. The relative expression of PDPN was calculated using the following formulae: i) $\Delta \mathrm{Cq}=\mathrm{Cq}_{\text {(target gene) }} \mathrm{Cq}_{\text {(reference gene) }}$; and ii) $\Delta \Delta \mathrm{Cq}=\Delta \mathrm{Cq}_{\text {(experiment) }}-\Delta \mathrm{Cq}_{\text {(control) }}$. Subsequently, the relative fold-change in gene expression was calculated as the $2^{-\Delta \Delta \mathrm{Cq}}$ value (16). Experiments were performed in triplicate.

IHC analysis. PDPN protein expression in paraffin-embedded osteosarcoma tissues $(n=168)$ and normal bone tissue specimens $(n=23)$ was determined by IHC analysis. Tissues were fixed prior to embedding in paraffin using $4 \%$ paraformaldehyde at room temperature for $6 \mathrm{~h}$. Briefly, tissue sections (thickness, $4 \mu \mathrm{m}$ ) were dewaxed using two washed with xylene (5 min each at room temperature) and dehydrated in a graded series of alcohol $(95,90,80$ and $70 \% ; 5$ min each at room temperature). Slides were prepared by boiling in citrate buffer for $5 \mathrm{~min}\left(95-100^{\circ} \mathrm{C}, \mathrm{pH} 6.0\right)$ prior to being cooled for $20 \mathrm{~min}$ at room temperature). In order to reduce non-specific antigen binding and prevent infection, slides were incubated with $0.2 \%$ trypsin in a $\mathrm{CO}_{2}$ incubator at $37^{\circ} \mathrm{C}$ for $50 \mathrm{~min}$. The slides were then incubated in $0.3 \%$ hydrogen peroxide for $3 \mathrm{~min}$ at room temperature to inhibit the activity of endogenous peroxidases. To reduce nonspecific binding, the slides were incubated in PBS supplemented with 10\% goat serum (Dako; Agilent Technologies, Inc.) at room temperature for $30 \mathrm{~min}$. The slides were subsequently incubated with monoclonal mouse antibodies against human PDPN (cat. no. D2-40; dilution, 1:100; Dako; Agilent Technologies, Inc.) overnight at $4^{\circ} \mathrm{C}$ in the refrigerator. The following day, slides were incubated with a goat anti-mouse antibody (cat. no. ZB-2305; 1:500; Histofine Simple Stain MAX PO-M; Nichirei Biosciences, Inc.) at room temperature for $30 \mathrm{~min}$. The slides were developed using 3,3'-diaminobenzidine solution. Slides were counterstained with hematoxylin and sealed with neutral gum. Negative controls were prepared by incubating with PBS instead of the primary antibody and were utilized to verify the immunostaining specificity. Light microscopy was used to observe the stained tissues $\mathrm{x} 100$ or $\mathrm{x} 400$ as indicated.

IHC assessment. The scoring system for PDPN expression was based on the percentage of positively stained tumor cells and the staining intensity. Initial scores for the percentage of positively stained cells were as follows: 0,$0 ; 1,1-25 ; 2,26-50$; and 3, 51-100\%. Staining intensity was scored as follows: 0, negative; 1 , weakly positive; 2 , moderately positive; and 3 , strongly positive. The immunostaining score, or immunoreactive score, was calculated as the product of the aforementioned two scores and was determined for all samples. IHC scoring was conducted in duplicate by two individual pathologists with extensive 
experience, that were blinded to the clinicopathological details of the patients and the identity of the slides. The percentage of positively stained cells in each sample was determined using $>5$ randomly selected fields of view (magnification, $x 400$ ). If different scoring results were reported by each pathologist, a third pathologist was consulted to reach a consensus regarding the final result. Scores ranged between 0 and 9, where 0-3 was considered to indicate low protein expression levels of PDPN, while scores of 4-9 represented high expression levels.

Statistical analysis. The SPSS statistical software package (version, 19.0; IBM Corp.) was used for statistical analysis of the data. The data were compared by one-way ANOVA followed by Dunnett's multiple comparisons post hoc test. All data are expressed as the means \pm standard deviation. A paired t-test was used to compare differences in expression levels in tumor and adjacent tissues. A $\chi^{2}$ test was utilized to investigate the association between clinicopathological features and PDPN expression. The Kaplan-Meier method was used to plot survival curves. Further analysis of the survival plots was achieved using the log-rank test. Univariate analysis was used to analyze differences between prognostic groups, and factors deemed significant by the univariate analysis were further analyzed by multivariate analysis. $\mathrm{P}<0.05$ was considered to indicate a statistically significant difference.

\section{Results}

PDPN mRNA expression levels are increased in human osteosarcoma tissues and cell lines. RT-qPCR analysis was employed to assess PDPN expression levels in human osteosarcoma tissue samples and cell lines. As shown in Fig. 1A, PDPN mRNA levels were observed to be higher in osteosarcoma cell lines compared with the normal hFOB 1.19 cell line. In addition, comparison of PDPN expression in 20 pairs of fresh POT and matched NCBT samples revealed significantly higher mRNA expression levels of PDPN in POT samples compared with in NCBTs $(\mathrm{P}<0.001$; Fig. 1B).

Increased mRNA expression levels of PDPN in patients with osteosarcoma and PM. In order to verify the results, PDPN mRNA levels in the 20 pairs of osteosarcoma and matched adjacent normal tissue samples were analyzed, noting that 8 of these patients presented PM. RT-qPCR analysis demonstrated higher PDPN expression in the osteosarcoma tissues in 6 out of the 8 cases with PM (Fig. 2A), and statistical significance was reached when the high and low expressors in the $\mathrm{PM}^{+}$group were compared $(\mathrm{t}=2.546, \mathrm{P}=0.014)$. Out of the remaining 12 patients with osteosarcoma and without PM, high levels of PDPN expression were observed in four patients (Fig. 2B); however, no significant difference between the high and low expressors in the $\mathrm{PM}^{-}$group was observed $(\mathrm{t}=0.495, \mathrm{P}=0.749)$. The difference between the $\mathrm{PM}^{+}$and $\mathrm{PM}^{-}$groups regarding PDPN mRNA expression of tissue samples was calculated. The results revealed that the PDPN mRNA expression in the $\mathrm{PM}^{+}$group was significantly higher compared with the $\mathrm{PM}^{-}$group. The mRNA expression levels of PDPN in the eight patients with $\mathrm{PM}^{+}$osteosarcoma and in the 12 patients with $\mathrm{PM}^{-}$osteosarcoma were significantly different $(\mathrm{P}<0.001$; Fig. 3).

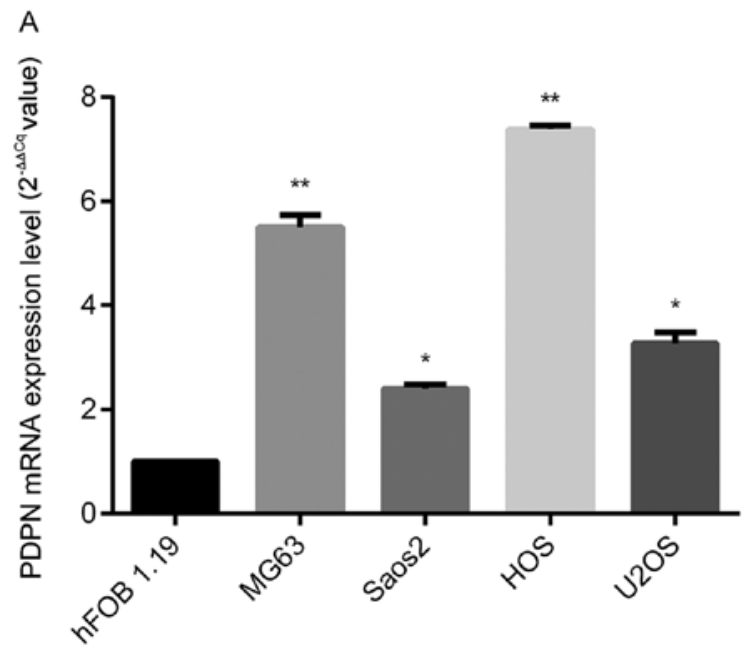

B

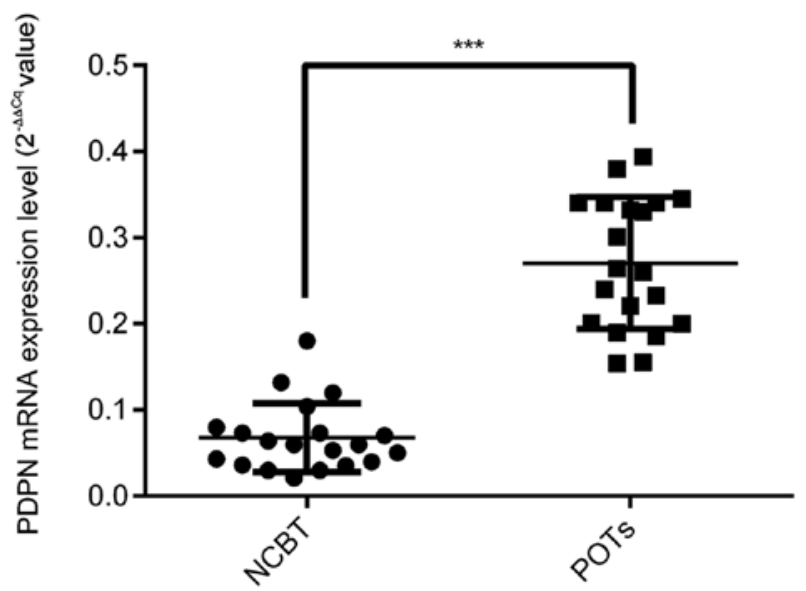

Figure 1. Increased PDPN mRNA expression in human osteosarcoma tissues and cell lines. (A) High mRNA expression levels of PDPN were observed in MG63, Saos2, HOS and U2OS cell lines compared with hFOB 1.19 cells ${ }^{*} \mathrm{P}<0.05{ }^{* *} \mathrm{P}<0.01$ compared with hFOB 1.19 cells. (B) PDPN mRNA levels were higher in POTs compared with the corresponding NCBTs. ${ }^{* * *} \mathrm{P}<0.001$. NCBT, non-cancerous bone tissues; PDPN, podoplanin; POTs, primary osteosarcoma tissues.

Association between PDPN expression and clinical parameters in patients with primary osteosarcoma. IHC analysis was used to determine PDPN expression in samples collected from 168 patients with osteosarcoma (Fig. 4). Significantly higher levels of PDPN expression were recorded in POTs $(n=74 / 168$; $44.0 \%)$ compared with the NCBTs $(n=2 / 23 ; 8.7 \%$; $P=0.009)$. Specific analysis of the association between PDPN expression in POTs and the clinical features of patients (Table I) revealed a significant association between high PDPN expression and Enneking stage $(\mathrm{P}<0.001)$ and $\mathrm{PM}(\mathrm{P}<0.001)$; however, no significant association was observed with patient age $(\mathrm{P}=0.196)$, sex $(\mathrm{P}=0.173)$, maximum tumor diameter $(\mathrm{P}=0.713)$, preoperative chemotherapy $(\mathrm{P}=0.635)$ and ALP levels in preoperative serum samples $(\mathrm{P}=0.119)$.

Univariate and multivariate analyses of clinical outcome prediction for patients with osteosarcoma. In univariate analysis, a significant association between overall survival and tumor size [hazard ratio $(\mathrm{HR})=2.185 ; 95 \% \mathrm{CI}=1.506-3.130$; $\mathrm{P}<0.001]$, Enneking stage $(\mathrm{HR}=3.476 ; 95 \% \mathrm{CI}=2.438-4.859$; 

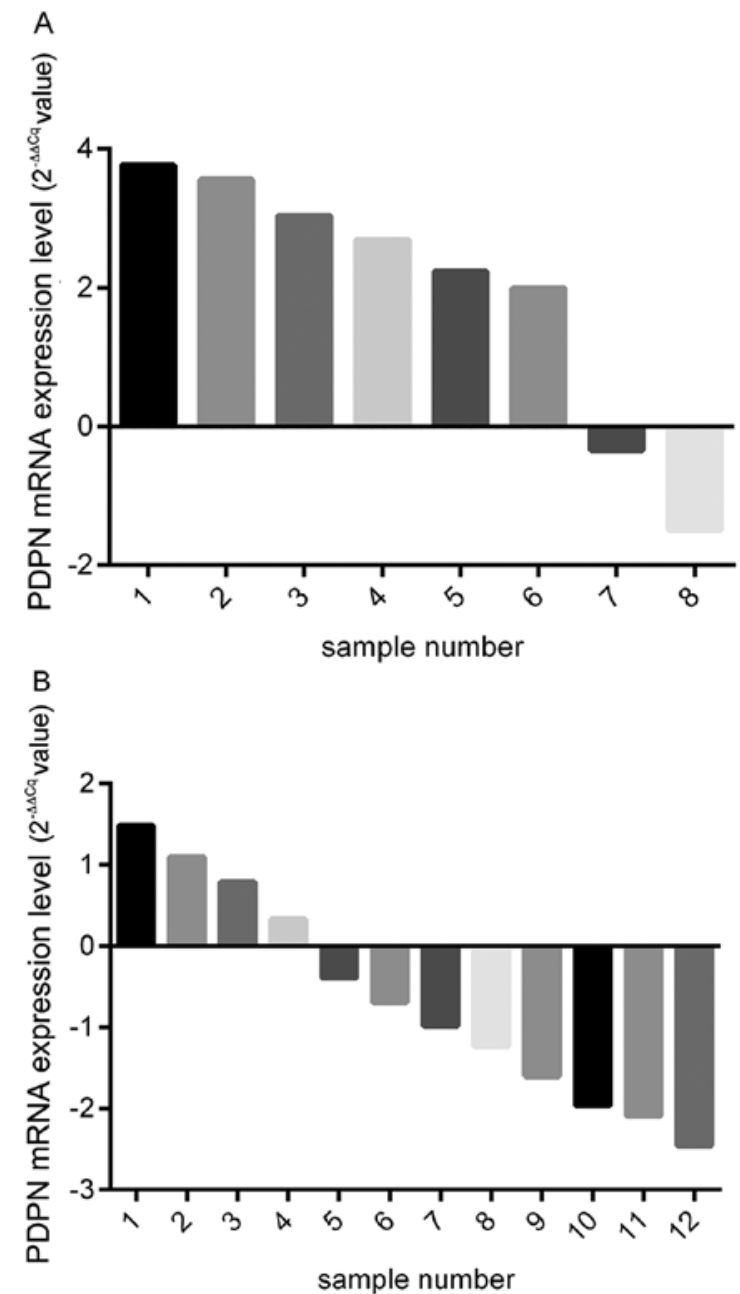

Figure 2. PDPN mRNA expression in osteosarcoma tissues. (A) PDPN mRNA expression in osteosarcoma tissues from $\mathrm{PM}^{+}$patients and corresponding normal tissues. (B) PDPN mRNA expression levels in PM- osteosarcoma tissues and corresponding normal tissues. Each bar represents one patient. PDPN, podoplanin; PM, pulmonary metastasis.

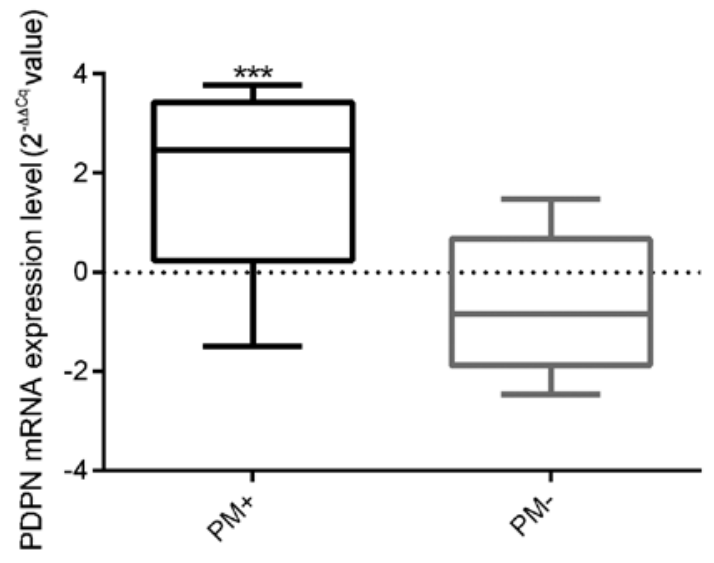

Figure 3. PDPN mRNA levels in osteosarcoma tissues from patients in the $\mathrm{PM}^{+}$and $\mathrm{PM}^{-}$groups. ${ }^{* * *} \mathrm{P}<0.001$. PDPN, podoplanin; PM, pulmonary metastasis.

$\mathrm{P}<0.001)$, $\mathrm{PM}(\mathrm{HR}=4.369 ; 95 \% \mathrm{CI}=2.891-6.338 ; \mathrm{P}<0.001)$ and PDPN expression $(\mathrm{HR}=1.933 ; 95 \% \mathrm{CI}=1.302-2.540 ; \mathrm{P}<0.001)$ was observed. In addition, Enneking stage $(\mathrm{HR}=1.718$;
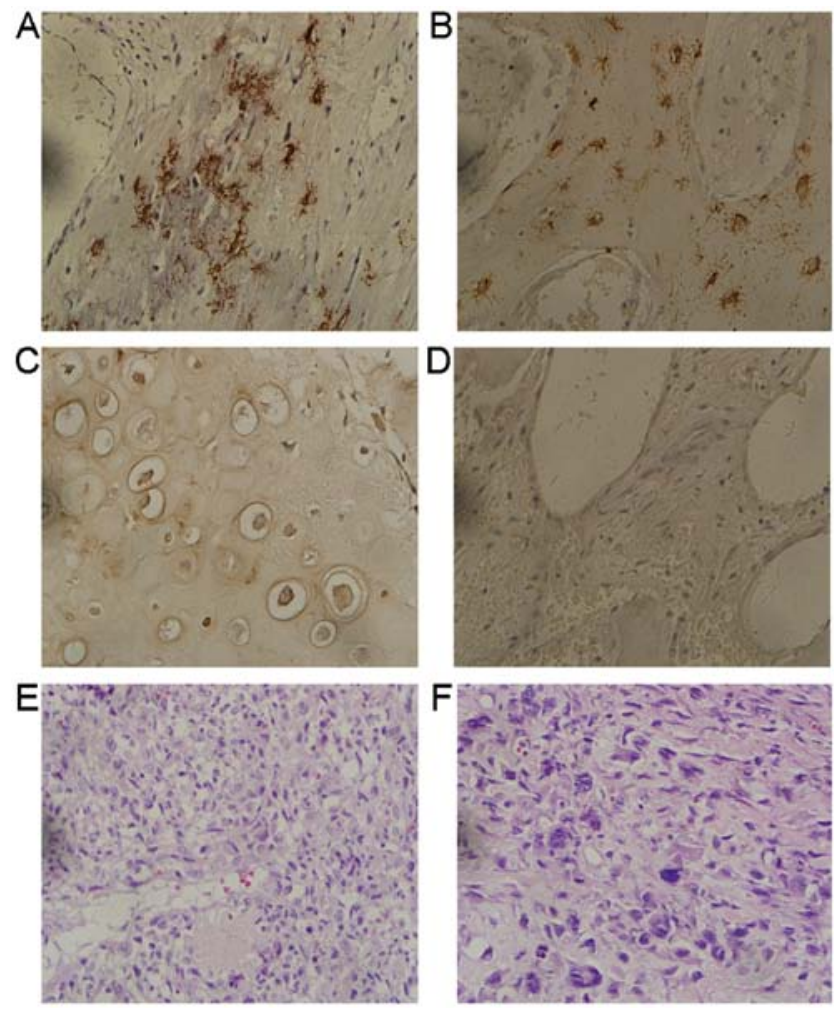

Figure 4. Protein expression levels of PDPN in paraffin-embedded osteosarcoma tissues. (A) High protein expression levels of PDPN in osteoblastic osteosarcoma (IHC staining score $=9$, high expression). (B) High protein expression levels of PDPN in telangiectasia osteosarcoma (IHC staining score $=9$, high expression). (C) High protein expression levels of PDPN in chondroblastic osteosarcoma (IHC staining score $=6$, high expression). (D) Low protein expression levels of PDPN in telangiectasia osteosarcoma (IHC staining score $=0$, low expression). (E) Hematoxylin-eosin staining of high expression group in osteoblastic osteosarcoma tissues. (F) Hematoxylin-eosin staining of high expression group in chondroblastic osteosarcoma tissues. All images were captured at $\mathrm{x} 400$ magnification and under identical microscopy conditions. IHC, immunohistochemistry; PDPN, podoplanin.

95\% $\mathrm{CI}=1.116-2.650 ; \mathrm{P}=0.013)$ and $\mathrm{PM}(\mathrm{HR}=3.164 ; 95 \%$ $\mathrm{CI}=1.817-5.413 ; \mathrm{P}<0.001)$ were identified as significant factors by multivariate analysis (Table II). In the prognostic analysis, there was no statistical difference identified between the high PDPN expression group and the low PDPN expression group ( $\mathrm{P}=0.683$; Fig. 5). Notably, significantly shorter overall survival rates for patients with Enneking stage III were observed compared with those with Enneking stage II ( $\mathrm{P}=0.013$; Fig. 6A), as well as for patients with $\mathrm{PM}$ compared with those without $(\mathrm{P}<0.001$; Fig. $6 \mathrm{~B})$, as determined using Kaplan-Meier analysis and the log-rank test.

\section{Discussion}

PM is the most reliable prognostic indicator for patients with resectable osteosarcoma, followed by Enneking stage, surgical complications, jumping lesions and local recurrence (17-19). Therefore, accurate assessment of PM is important for predicting patient prognosis and developing effective surgical treatment plans. A previous study demonstrated that positron emission tomography-computed tomography (CT) is a valuable tool for the detection of PM in patients with osteosarcoma when PM is suspected (20). However, tumors $<0.2 \mathrm{~cm}$ in 
Table I. Association between PDPN protein expression and the clinicopathological characteristics of patients with osteosarcoma.

\begin{tabular}{|c|c|c|c|c|}
\hline \multirow[b]{2}{*}{ Clinical parameters } & \multicolumn{2}{|c|}{ PDPN, n (\%) } & \multirow[b]{2}{*}{$\chi^{2}$} & \multirow[b]{2}{*}{ P-value } \\
\hline & High expression & Low expression & & \\
\hline Age, years & & & 1.904 & 0.196 \\
\hline$\leq 25$ & $43(39.8)$ & $65(60.2)$ & & \\
\hline$>25$ & $31(51.7)$ & $29(48.3)$ & & \\
\hline Sex & & & 1.857 & 0.173 \\
\hline Male & $45(45.9)$ & $53(54.1)$ & & \\
\hline Female & $29(41.4)$ & $41(58.6)$ & & \\
\hline Tumor diameter, $\mathrm{cm}$ & & & 0.015 & 0.713 \\
\hline$\leq 5$ & $32(42.1)$ & $44(57.9)$ & & \\
\hline$>5$ & $42(45.7)$ & $50(54.3)$ & & \\
\hline Enneking stage & & & 9.805 & $<0.001$ \\
\hline I and II & $56(38.6)$ & $89(61.4)$ & & \\
\hline III & $18(78.3)$ & $5(21.7)$ & & \\
\hline Preoperative chemotherapy & & & 0.159 & 0.635 \\
\hline Yes & $23(65.7)$ & $12(34.3)$ & & \\
\hline No & $51(38.3)$ & $82(61.7)$ & & \\
\hline Preoperative serum ALP & & & 2.217 & 0.119 \\
\hline High & $43(45.3)$ & $52(54.7)$ & & \\
\hline Normal & $31(42.5)$ & $42(57.5)$ & & \\
\hline PM & & & 9.805 & $<0.001$ \\
\hline Yes & $18(78.3)$ & $5(21.7)$ & & \\
\hline No & $56(38.6)$ & $89(61.4)$ & & \\
\hline
\end{tabular}

ALP, alkaline phosphatase; PM, pulmonary metastasis.

size in the lung are beyond the limit of detection, therefore CT imaging has limited sensitivity for the early detection of PM (5). High accuracy, preoperative assessment of PM is a vital part of osteosarcoma treatment. To the best of our knowledge, There are no studies that have investigated the association between PDPN expression and PM in patients with osteosarcoma.

In the present study, the mRNA expression levels of PDPN in human osteosarcoma tissues and four cell lines and its association with osteosarcoma prognosis were investigated. Consistent with a previous report (7), increased PDPN expression was observed in human osteosarcoma tissues and the same four cell lines. Comparison of PDPN expression in 20 pairs of fresh POT and matched NCBT samples demonstrated significantly higher levels of PDPN mRNA in POT samples compared with NCBTs. In addition, the present study demonstrated that PDPN expression was significantly higher in patients with osteosarcoma with PM compared with in patients without PM. Furthermore, high PDPN expression was significantly associated with Enneking stage and PM. The major difference between Enneking stages III and II is metastasis to a distant organ. In osteosarcoma, the most common metastatic site is the lung. The results of the present study demonstrated that high PDPN expression is associated with PM, which suggested that increased PDPN expression may function as a marker of disease progression, whereby the tumor cells pass through the interventricular barrier, enter the blood stream and metastasize to the lungs.

High PDPN expression has been previously reported in several types of cancer and is used as an effective biomarker of tumor malignancy and prognosis (21-23). A previous study confirmed that anti-PDPN monoclonal antibodies serve an inhibitory role in PDPN-expressing tumors in terms of their growth and hematogenous metastasis (24). A definite association between high PDPN expression and PDPN-mediated lung metastasis in osteosarcoma has not been established in previous studies. Thus far, several hypotheses have been proposed to explain the effect of PDPN on promoting tumor metastasis, including accelerating epithelial-mesenchymal transition (EMT) (25), inducing collective cell migration (26), inducing platelet activation and aggregation (27-29), and enhancing lymphangiogenesis (30). Regarding the mechanism of PDPN in mediating PM in patients with osteosarcoma, the following hypothesis is speculated based on previous studies (25,27-29): i) The assumption that PDPN and EMT processes are associated; ii) PDPN may serve as an endogenous ligand for C-type lectin-like receptor-2 during tumor metastasis; and iii) PDPN may promote platelet-specific acceleration of PM to some extent. Overall, further research to understand the mechanisms underlying PDPN-mediated PM is required in order to develop more effective treatment strategies for patients with osteosarcoma. 
Table II. Univariate and multivariate analysis of prognostic factors for patients with osteosarcoma.

\begin{tabular}{|c|c|c|c|c|c|c|}
\hline Variables & HR & Univariate $95 \% \mathrm{CI}$ & P-value & HR & Multivariate $95 \% \mathrm{CI}$ & $\mathrm{P}$-value \\
\hline \multicolumn{7}{|l|}{ Age (years) } \\
\hline$\leq 25$ vs. $>25$ & 1.297 & $0.926-1.383$ & 0.343 & & & \\
\hline \multicolumn{7}{|l|}{ Sex } \\
\hline Female vs. male & 1.165 & $0.816-1.407$ & 0.175 & & & \\
\hline \multicolumn{7}{|c|}{ Tumor diameter, cm } \\
\hline$>5$ vs. $\leq 5$ & 2.185 & $1.506-3.130$ & $<0.001$ & 1.229 & $0.927-1.853$ & 0.143 \\
\hline \multicolumn{7}{|l|}{ Enneking stage } \\
\hline III vs. I/II & 3.476 & $2.438-4.859$ & $<0.001$ & 1.718 & $1.116-2.650$ & 0.013 \\
\hline \multicolumn{7}{|c|}{ Preoperative Chemotherapy } \\
\hline No vs. yes & 1.152 & $0.667-1.572$ & 0.498 & & & \\
\hline \multicolumn{7}{|c|}{ Preoperative serum ALP } \\
\hline High vs. normal & 1.263 & $0.889-1.784$ & 0.627 & & & \\
\hline \multicolumn{7}{|l|}{ PM } \\
\hline Yes vs. no & 4.369 & $2.891-6.338$ & $<0.001$ & 3.164 & $1.817-5.413$ & $<0.001$ \\
\hline \multicolumn{7}{|l|}{ Podoplanin } \\
\hline High vs. low & 1.933 & $1.302-2.540$ & $<0.001$ & 1.122 & $0.830-1.458$ & 0.683 \\
\hline
\end{tabular}

ALP, alkaline phosphatase; CI, confidence interval; HR, hazard ratio; PM pulmonary metastasis.

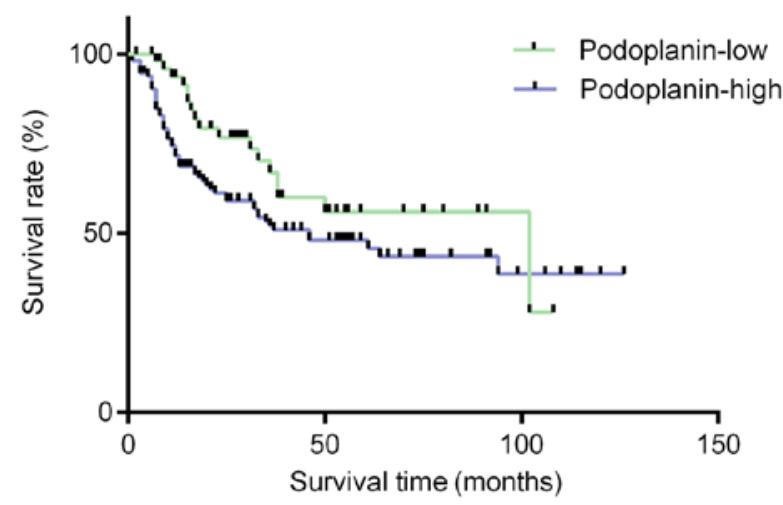

Figure 5. Kaplan-Meier analysis of overall survival of patients with osteosarcoma based on podoplanin expression $(\mathrm{P}=0.683)$.

Kunita et al (7) reported that the expression of PDPN in MG63, HOS and U2OS osteosarcoma cell lines was able to induce platelet aggregation. Treatment with PDPN small interfering RNA or specific neutralizing antibodies inhibited PDPN expression (7). Enhanced migration of Dunn osteosarcoma cells was observed following overexpression of PDPN, while cell proliferation remained unaffected. The present study observed increased PDPN expression in patients with osteosarcoma and PM. Furthermore, the difference in PDPN mRNA expression levels of tissue samples between the $\mathrm{PM}^{+}$ group and $\mathrm{PM}^{-}$group was calculated. The results demonstrated that the PDPN mRNA expression in the $\mathrm{PM}^{+}$group was significantly higher compared with the $\mathrm{PM}^{-}$group. The differences in PDPN mRNA expression levels between the eight patients with osteosarcoma with $\mathrm{PM}$ and the 12 patients without PM were statistically significant $(\mathrm{P}<0.001)$. Taking the results of all studies into consideration, it is possible
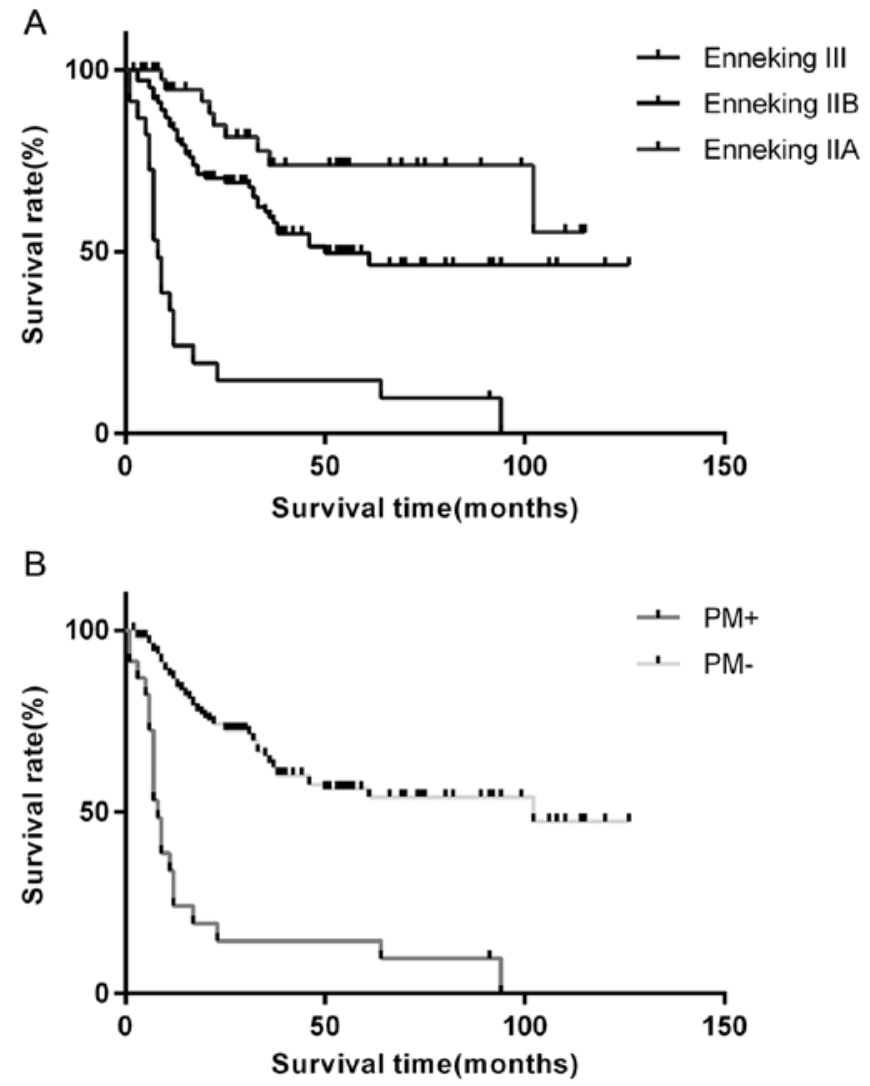

Figure 6. Kaplan-Meier analysis of overall survival in patients with osteosarcoma. Patients were divided into groups based on (A) Enneking stage $(\mathrm{P}=0.013)$ and $(\mathrm{B}) \mathrm{PM}(\mathrm{P}<0.001)$. $\mathrm{PM}$, pulmonary metastasis.

that PDPN may serve an important role in mediating tumor metastasis in patients with osteosarcoma, and that high PDPN 
expression may be involved in the development of PM in these patients. This is supported by the observation that PDPN expression was identified as a predictor of PM in patients with osteosarcoma in the present study. Increased PDPN expression may therefore serve as an effective and novel predictor of PM in patients with osteosarcoma in the clinic. However, this requires confirmation in a larger cohort of patients.

The association between ALP levels and the prognosis of patients with osteosarcoma has been investigated in numerous previous studies (31-33); however, no formal consensus has been reached. According to a recent meta-analysis (34), increased ALP levels are associated with reduced overall survival rates in patients with osteosarcoma, and ALP serves as a biomarker. These results are inconsistent with those of the present study, where no significant association between these factors was observed. In addition, no association between preoperative chemotherapy and patient prognosis was observed in a previous study (35). It is possible that preoperative chemotherapy reduces the extent of tumor edema, which enables clear observation of the tumor boundary and the complete removal of the tumor. However, it may not significantly impact the survival of patients with osteosarcoma.

In the present study, a trend was observed for high PDPN expression to be involved in worse outcome, but this was not deemed to be significant using Kaplan Meier analysis. PDPN was significantly associated with PM, and PM was an independent prognostic factor. Therefore, future studies with larger cohorts are required to confirm whether PDPN alone can be an independent prognostic factor for osteosarcoma. A previous study demonstrated that PDPN immunoreactivity in tumor cells may be an effective indicator of poor prognosis for patients with non-small cell lung cancer (21), which is consistent with the results of the present study. In addition, Enneking stage and PM were identified as independent prognostic markers in patients with osteosarcoma in the present study, which is consistent with previous studies $(36,37)$. Furthermore, an association between high PDPN expression levels and PM was observed, and PDPN expression was also identified as a significant prognostic marker, according to univariate analyses. Nevertheless, it was not an independent prognostic factor in osteosarcoma according to multivariate analysis. Therefore, it is possible that PDPN may mediate PM in patients with osteosarcoma, which subsequently affects their prognosis. PDPN is not an independent prognostic factor; however, the present study demonstrated that PDPN overexpression is associated with lung metastasis, and one may hypothesize that PDPN-induced lung metastasis will indirectly affect the prognosis of patients. Future studies with larger experimental samples will be required to explore the potential of PDPN expression as a prognostic marker. In addition, the results of the present study indicated that PDPN expression was increased in patients with primary osteosarcoma with PM, and a significant association with the risk of PM development. Therefore, enhanced PDPN expression was proposed as a molecular biomarker for PM development and the subsequent prognosis of patients with osteosarcoma.

The present study had several limitations. First, surgical specimens rather than biopsy specimens were analyzed; therefore, it is possible that demineralization or preoperative chemotherapy may have affected the results of PDPN immunostaining. Only the sample tissue at a certain time (surgical removal) was selected in the present study. This represented a single time point, not a continuous observation. In the present study, only differences between the $\mathrm{PM}^{+}$and $\mathrm{PM}^{-}$groups were observed. If possible, the effect of time factors on PDPN expression should be examined. Second, tumor size was recorded in the preoperative medical records or recorded in the surgical records. This resulted in subjectivity. In further studies, parameters should be recorded more objectively. Third, heterogeneity at the protein and mRNA levels is an important feature of malignant tumors, and the sample size in the present study was likely too small to accurately determine the differential expression of PDPN among the various pathological types. Therefore, additional experiments in a larger sample cohort are required.

In conclusion, high PDPN expression levels were observed to be significantly associated with PM in patients with osteosarcoma in the present study. PDPN expression may be a useful immunological marker for PM in patients with osteosarcoma. However, further experiments are required to confirm these results.

\section{Acknowledgements}

Not applicable.

\section{Funding}

The present study was supported by grants from The Science and Technology Talents Program of Harbin (Harbin, China; grant nos. 2014RFXGJ041 and 2014RFQGJ094), a post-doctoral fund (grant. no. 160780) and the Heilongjiang Natural Science Foundation (grant nos. QC2016102 and H2016002).

\section{Availability of data and materials}

The datasets used and analyzed during the present study are available from the corresponding author on reasonable request.

\section{Authors' contributions}

XW and QM designed the study and wrote the manuscript. JW and LN collected the specimen and patient data. XW, WL, JB, and QS performed the experiments and analyzed the data. All authors read and approved the final manuscript.

\section{Ethics approval and consent to participate}

The present study was performed in accordance with the Declaration of Helsinki and approved by the institutional Ethics Committee of Harbin Medical University and Harbin Medical University Cancer Hospital. Written informed consent was obtained from patients or their legal guardians.

\section{Patient consent for publication}

Not applicable.

\section{Competing interests}

The authors declare that they have no competing interests. 


\section{References}

1. Biazzo A and De Paolis M: Multidisciplinary approach to osteosarcoma. Acta Orthop Belg 82: 690-698, 2016

2. Biermann JS, Chow W, Reed DR, Lucas D, Adkins DR Agulnik M, Benjamin RS, Brigman B, Budd GT, Curry WT, et al: NCCN guidelines insights: Bone cancer, version 2.2017. J Natl Compr Canc Netw 15: 155-167, 2017.

3. Nataraj V, Batra A, Rastogi S, Khan SA, Sharma MC, Vishnubhatla $\mathrm{S}$ and Bakhshi S: Developing a prognostic model for patients with localized osteosarcoma treated with uniform chemotherapy protocol without high dose methotrexate: A single-center experience of 237 patients. J Surg Oncol 112: 662-668, 2015.

4. Boye K, Del Prever AB, Eriksson M, Saeter G, Tienghi A, Lindholm P, Fagioli F, Skjeldal S, Ferrari S and Hall KS: High-dose chemotherapy with stem cell rescue in the primary treatment of metastatic and pelvic osteosarcoma: Final results of the ISG/SSG II study. Pediatr Blood Cancer 61: 840-845, 2014.

5. Heaton TE, Hammond WJ, Farber BA, Pallos V, Meyers PA, Chou AJ, Price AP and LaQuaglia MP: A 20-year retrospective analysis of CT-based pre-operative identification of pulmonary metastases in patients with osteosarcoma: A single-center review. J Pediatr Surg 52: 115-119, 2017.

6. Oki H, Kaneko MK, Ogasawara S, Tsujimoto Y, Liu X, Sugawara M, Takakubo Y, Takagi M and Kato Y: Characterization of monoclonal antibody LpMab-7 recognizing Non-PLAG domain of podoplanin. Monoclon Antib Immunodiagn Immunother 34: 174-180, 2015.

7. Kunita A, Kashima TG, Ohazama A, Grigoriadis AE and Fukayama M: Podoplanin is regulated by AP-1 and promotes platelet aggregation and cell migration in osteosarcoma. Am J Pathol 179: 1041-1049, 2011.

8. Kato Y, Kaneko MK, Kuno A, Uchiyama N, Amano K, Chiba Y, Hasegawa Y, Hirabayashi J, Narimatsu H, Mishima K and Osawa M: Inhibition of tumor cell-induced platelet aggregation using a novel anti-podoplanin antibody reacting with its platelet-aggregation-stimulating domain. Biochem Biophys Res Commun 349: 1301-1307, 2006.

9. Miyata K, Takemoto A, Okumura S, Nishio M and Fujita N: Podoplanin enhances lung cancer cell growth in vivo by inducing platelet aggregation. Sci Rep 7: 4059, 2017.

10. Takagi S, Sato S, Oh-hara T, Takami M, Koike S, Mishima Y, Hatake K and Fujita N: Platelets promote tumor growth and metastasis via direct interaction between Aggrus/podoplanin and CLEC-2. PLoS One 8: e73609, 2013.

11. Swain N, Kumar SV, Routray S,Pathak J and Patel S: Podoplanin-a novel marker in oral carcinogenesis. Tumour Biol 35: 8407-8413, 2014.

12. Yamaki E, Yajima T, Kosaka T, Mogi A, Tanaka S and Kuwano H: Podoplanin overexpression in human mesothelioma cell lines enhances the tumorigenic phenotype. Oncol Rep 29: 932-940, 2013.

13. Kaneko MK, Oki H, Ogasawara S, Takagi $M$ and Kato $Y$ : Anti-podoplanin monoclonal antibody LpMab-7 detects metastatic lesions of osteosarcoma. Monoclon Antib Immunodiagn Immunother 34: 154-161, 2015.

14. DePaolo CJ, Foster WS, Dabezies EJ and D'Ambrosia RD: A case report of malignant mesenchymoma with discussion of musculoskeletal tumor staging: The Enneking system. Orthopedics 11: 1263-1276, 1988.

15. Jo VY and Doyle LA: Refinements in sarcoma classification in the current 2013 world health organization classification of tumours of soft tissue and bone. Surg Oncol Clin N Am 25: 621-643, 2016.

16. Livak KJ and Schmittgen TD: Analysis of relative gene expression data using real-time quantitative PCR and the 2(-Delta Delta C(T)) method. Methods 25: 402-408, 2001.

17. Grimer RJ, Aydin BK, Wafa H, Carter SR, Jeys L, Abudu A and Parry M: Very long-term outcomes after endoprosthetic replacement for malignant tumours of bone. Bone Joint J 98-B: 857-864, 2016

18. Koirala P, Roth ME, Gill J, Chinai JM, Ewart MR, Piperdi S, Geller DS, Hoang BH, Fatakhova YV, Ghorpade M, et al: HHLA2, a member of the B7 family, is expressed in human osteosarcoma and is associated with metastases and worse survival. Sci Rep 6: 31154, 2016.

19. Puri A, Byregowda S, Gulia A, Crasto S and Chinaswamy G: A study of 853 high grade osteosarcomas from a single institution-Are outcomes in Indian patients different? J Surg Oncol 117: 299-306, 2018.
20. Angelini A, Ceci F, Castellucci P, Graziani T, Polverari G, Trovarelli G, Palmerini E, Ferrari S, Fanti S and Ruggieri P: The role of ${ }^{18} \mathrm{~F}$-FDG PET/CT in the detection of osteosarcoma recurrence. Eur J Nucl Med Mol Imaging 44: 1712-1720, 2017.

21. Kadota K, Huang CL, Liu D, Nakashima N, Yokomise $H$, Ueno $\mathrm{M}$ and Haba R: The clinical significance of the tumor cell D2-40 immunoreactivity in non-small cell lung cancer. Lung Cancer 70: 88-93, 2010.

22. Yuan P, Temam S, El-Naggar A, Zhou X, Liu DD, Lee JJ and Mao L: Overexpression of podoplanin in oral cancer and its association with poor clinical outcome. Cancer 107: 563-569, 2006.

23. Minardi D, d'Anzeo G, Lucarini G, Filosa A, Zizzi A, Simonetti O, Polito M Jr, Offidani AM, Di Primio R, Montironi R and Muzzonigro G: D2-40 immunoreactivity in penile squamous cell carcinoma: A marker of aggressiveness. Hum Pathol 42: 1596-1602, 2011.

24. Nakazawa Y, Takagi S, Sato S, Oh-hara T, Koike S, Takami M, Arai $\mathrm{H}$ and Fujita N: Prevention of hematogenous metastasis by neutralizing mice and its chimeric anti-Aggrus/podoplanin antibodies. Cancer Sci 102: 2051-2057, 2011.

25. Martin-Villar E, Megias D, Castel S, Yurrita MM, Vilaró S and Quintanilla M: Podoplanin binds ERM proteins to activate RhoA and promote epithelial-mesenchymal transition. J Cell Sci 119: 4541-4553, 2006.

26. Wicki A, Lehembre F, Wick N, Hantusch B, Kerjaschki D and Christofori G: Tumor invasion in the absence of epithelial-mesenchymal transition: Podoplanin-mediated remodeling of the actin cytoskeleton. Cancer Cell 9: 261-272, 2006.

27. Kunita A, Kashima TG, Morishita Y, Fukayama M, Kato Y, Tsuruo $\mathrm{T}$ and Fujita $\mathrm{N}$ : The platelet aggregation-inducing factor aggrus/podoplanin promotes pulmonary metastasis. Am J Pathol 170: 1337-1347, 2007.

28. Suzuki-Inoue K, Kato Y, Inoue O, Kaneko MK, Mishima K, Yatomi Y, Yamazaki Y, Narimatsu H and Ozaki Y: Involvement of the snake toxin receptor CLEC-2, in podoplanin-mediated platelet activation, by cancer cells. J Biol Chem 282: 25993-26001, 2007.

29. Nakazawa Y, Sato S, Naito M, Kato Y, Mishima K, Arai H, Tsuruo $T$ and Fujita N: Tetraspanin family member CD9 inhibits Aggrus/podoplanin-induced platelet aggregation and suppresses pulmonary metastasis. Blood 112: 1730-1739, 2008.

30. Cueni LN, Hegyi I, Shin JW, Albinger-Hegyi A, Gruber S, Kunstfeld R, Moch H and Detmar M: Tumor lymphangiogenesis and metastasis to lymph nodes induced by cancer cell expression of podoplanin. Am J Pathol 177: 1004-1016, 2010.

31. Han J, Yong B, Luo C, Tan P, Peng T and Shen J: High serum alkaline phosphatase cooperating with MMP-9 predicts metastasis and poor prognosis in patients with primary osteosarcoma in Southern China. World J Surg Oncol 10: 37, 2012.

32. Kim SH, Shin KH, Moon SH, Jang J, Kim HS, Suh JS and Yang WI: Reassessment of alkaline phosphatase as serum tumor marker with high specificity in osteosarcoma. Cancer Med 6: 1311-1322, 2017.

33. Marais LC, Bertie J, Rodseth R, Sartorius B and Ferreira N: Pre-treatment serum lactate dehydrogenase and alkaline phosphatase as predictors of metastases in extremity osteosarcoma. J Bone Oncol 4: 80-84, 2015.

34. Hao H, Chen L, Huang D, Ge J, Qiu Y and Hao L: Meta-analysis of alkaline phosphatase and prognosis for osteosarcoma. Eur $\mathrm{J}$ Cancer Care (Engl) 26, 2017.

35. Nagano A, Ishimaru D, Nishimoto Y, Akiyama $\mathrm{H}$ and Kawai A: Primary bone sarcomas in patients over 40 years of age: A retrospective study using data from the bone tumor registry of Japan. J Orthop Sci 22: 749-754, 2017.

36. Lisenda L, Linda ZA, Snyman FPJ, Kyte RD and Lukhele M: Osteosarcoma patient outcomes at a South African tertiary hospital. S Afr Med J 107: 754-757, 2017.

37. Zhang B, Pang QJ, Zhang HJ and Yuan Y: Multivariate analysis for prognostic factors among 43 patients with osteosarcoma. Zhongguo Gu Shang 24: 982-986, 2011 (In Chinese).

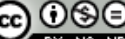

This work is licensed under a Creative Commons Attribution-NonCommercial-NoDerivatives 4.0 International (CC BY-NC-ND 4.0) License. 